\title{
Universal screening for SARS-CoV-2 before labor admission during Covid-19 pandemic in Madrid
}

https://doi.org/10.1515/jpm-2020-0236

Received May 27, 2020; accepted July 1, 2020; published online July 20, 2020

\section{Abstract}

Objectives: Asymptomatic women admitted to labor may act as silent spreaders of COVID-19. Therefore, universal screening at admission has been proposed. The objective of the study was to evaluate the performance of universal screening for SARS-CoV-2 using quantitative reverse transcription polymerase-chain-reaction (qRT-PCR) tests in women admitted to labor.

Methods: Observational retrospective study of a cohort of pregnant women admitted to labor and delivery between April 8 and May 2, 2020 in a large maternity in Madrid. SARS-CoV-2 screening with qRT-PCR from combined nasopharyngeal and oropharyngeal swabs was carried out systematically. Screening performance was described.

Results: We attended 212 deliveries. Nine cases with COVID19 diagnosis before admission were excluded. In the remaining 203 women, seven referred COVID-19-related symptoms but only one had a positive qRT-PCR. Among the 194 asymptomatic women, only one case $(0.5 \%)$ was positive. Conclusions: The percentage of positive tests in asymptomatic women admitted to delivery was only $0.5 \%$ during the post-peak period.

*Corresponding author: Ignacio Herraiz, Fetal Medicine Unit Maternal and Child Health and Development Network (Red SAMID-RD12/0026/0016), Department of Obstetrics and Gynecology, Hospital Universitario 12 de Octubre, Instituto de Investigación Hospital 12 de Octubre (imas12), Universidad Complutense de Madrid, Avda. Córdoba s/n, Madrid 28041, Spain, Phone: +(0)34 13908310 , E-mail: ignacio.herraiz@salud.madrid.org. https://orcid.org/00000001-6807-4944

Dolores Folgueira and Rafael Delgado: Department of Clinical Microbiology, Hospital Universitario 12 de Octubre, Instituto de Investigación Hospital 12 de Octubre (imas12), Universidad Complutense de Madrid, Madrid, Spain

Cecilia Villalaín, Laura Forcén and Alberto Galindo: Fetal Medicine Unit - Maternal and Child Health and Development Network (Red SAMID-RD12/0026/0016), Department of Obstetrics and Gynecology, Hospital Universitario 12 de Octubre, Instituto de Investigación Hospital 12 de Octubre (imas12), Universidad Complutense de Madrid, Madrid, Spain
Keywords: admission; COVID-19; pregnancy; SARS-CoV-2; screening.

\section{Introduction}

The recent outbreak of COVID-19 has posed a reorganizing challenge for health services. This is especially true for those cities like Madrid that have suffered a more sudden evolution of the pandemic, together with the lack of implementation of massive diagnostic testing, collapse of intensive care units and a restriction of appropriate protective equipment.

Maternity hospitals are not immune to this problem and have been forced to take adaptive measures. Pregnant women are a relatively low risk group for COVID-19 since they are generally young and healthy, who usually suffer from pauci- or asymptomatic courses. Furthermore, it has not been proven that pregnancy worsens the prognosis of the disease [1]. However, pregnant women are great frequent users of health centers and may inadvertently spread the disease, this being most relevant at the time of delivery assistance. Our first case of COVID-19 was diagnosed on March 3rd by quantitative reverse transcription polymerase-chain-reaction (qRT-PCR) test, in the fifth day after caesarean section, being asymptomatic at admission. During the most intense phase of the pandemic, we also had other cases of asymptomatic women who were admitted for non-COVID-19-related conditions that soon after developed symptoms with the consequent risk of contagion. Thus, from a very early stage, it was our feeling that it was important to screen pregnant women admitted to labor. However, the prioritization of resources did not make it possible to implement this strategy at first. From March 31st to April 7th, it was decided to perform qRT-PCR testing to all pregnant women with a scheduled cesarean section or labor induction. Seventeen tests were performed in asymptomatic and three cases were positive (17.6\%). Given this experience, it was decided to extend the PCR test to all pregnant women who were admitted for labor since April 8th, when the rate of infection had already begun a rapid decline. 


\section{Objective}

The objective of the study was to evaluate the performance of universal screening for SARS-CoV-2 using qRT-PCR tests in women admitted to labor during the COVID-19 pandemic in Madrid, and compare it with the results described in other locations at different times of the pandemic.

\section{Materials and methods}

This is an observational retrospective cohort study conducted in our tertiary hospital located in the south of Madrid between April 8th and May 2nd, 2020. Pregnant women admitted to labor and delivery were included. Those with COVID-19 diagnosed before admission were excluded. At the time of admission, women were questioned about COVID-19-related symptoms, combined nasopharyngeal and oropharyngeal swabs were systematically collected and a qRT-PCR test for SARS-CoV-2 was performed. For women with scheduled procedures, including cesarean sections and induction of labor, the test was performed within the last $48 \mathrm{~h}$ before admission. If they were found positive, the option of delaying the scheduled delivery was individually discussed with the patient if there were no contraindications for such delay. Women were allowed to be accompanied by one birth partner during labor, birth and immediate postpartum period. This birth partner was not routinely screened by qRT-PCR, but was only suitable for accompaniment in the absence of COVID19-related symptoms and provided that promised to stay in the room, keep social distancing measures, use hand sanitizer gel regularly and wear a mask. The exception for such accompaniment was cesarean section since the partner was not screened by qRT-PCR and given the higher risk of potential spread of SARS-CoV-2 in the operating environment in case of being positive. In case the woman was found positive before delivery, maternal reassessment was performed including temperature measurement, blood pressure, respiratory rate, and oxygen saturation to assure the absence of clinical deterioration. Moreover, isolation measures were taken: all the involved healthcare staff used personal protective equipment, the number of staff members entering the room was minimized and delivery took place in a delivery room reserved for SARS-CoV-2 positive cases. Fetal monitoring and decisions regarding the mode of birth were maintained according to general protocols. After birth, newborns were tested for SARS-CoV-2 and thromboprophylaxis was initiated after $12 \mathrm{~h}$.

The study was approved by the local Research Ethics Committee and written informed consent was not required. We have complied with the World Medical Association Declaration of Helsinki regarding ethical conduct of research involving human subjects. We collected data about the presence of symptoms of COVID-19 at admission, including fever, cough, rhinorrhea, dyspnea, chest pain, diarrhea, myalgia or new anosmia or ageusia. If one or more were present, the patient was considered symptomatic. A case of COVID-19 was considered confirmed when qRT-PCR was positive for SARS-CoV-2, irrespective of the clinical status. If initial testing was negative but the symptoms were strongly suggestive of COVID-19, the woman was resampled. If still negative, the case was considered probable but not confirmed.
Samples were collected with flocked swabs in UTM ${ }^{\mathrm{TM}}$ viral transport medium (Copan Diagnostics, Brescia, Italy). Nucleic acid extraction was performed by using MicrolabStarlet IVD platform using the STARMag $96 \times 4$ Universal Cartridge Kit (Seegene, Seoul, South Korea) or NucliSENS EasyMAG instrument (bioMerieux, Marcy l'Etoile, France). Both platforms are commonly used in our Laboratory for the extraction of nucleic acids from respiratory samples. For qRT-PCR, we used LightCycler 480 System instrument II (Roche Life Science, Indianapolis, IN, USA), performing the test TaqMan 2019nCoV assay Kit v1, provided by Thermo Fisher Scientific (Waltham, Massachusetts, USA), that amplifies three different viral regions in singleplex reactions. Positive and negative controls, as well as internal control, were tested in each run. To confirm amplifications in late cycles, a previously published qRT-PCR protocol to detect E gene [2] was adapted for processing on the automated molecular diagnostic platform Panther Fusion, using its open access functionality [3] using an open access protocol for the Panther Fusion.

Screening results are presented together with the results of the three other studies (Sutton et al. [4], Vintzileos et al. [5], and Gagliardi et al. [6]) that have reported data on the universal screening of SARS-CoV-2 on admission to labor and delivery during the pandemic of COVID-19.

\section{Results}

We attended 212 deliveries during the study period. We excluded nine cases with COVID-19 diagnosis before admission to labor and delivery (6/9 were asymptomatic at delivery and only $1 / 6$ tested positive). In the study group of 203 women, seven referred COVID-19-related symptoms but only one had a positive qRT-PCR. This case also had associated preeclampsia with severity criteria, which was induced at week 35 and had an uncomplicated instrumental delivery with a good postnatal result. Among the 194 asymptomatic women, only one was positive and resulted in an uncomplicated term eutocic delivery. The results of our universal screening for SARS-CoV-2 in women admitted to labor and delivery in our and previous studies are summarized in Table 1.

\section{Discussion}

Our study showed a low positive rate of SARS-CoV-2 (0.5\%) in asymptomatic women screened with qRT-PCR at the time of admission to labor during the study period in Madrid. Our city suffered the effects of COVID-19 in a similar way to New York and Tuscany. However, the results of universal screening in women admitted to labor were remarkably different among centers. The most likely explanation relies in the chronology itself of the pandemic and the screening: while in New York the first obstetrical 
Table 1: Results of the universal screening for SARS-CoV-2 by quantitative reverse transcription polymerase-chain-reaction in our center and in other three studies published to date from other regions that have also been affected by Covid-19 pandemic.

\begin{tabular}{|c|c|c|c|c|c|}
\hline \multicolumn{2}{|l|}{ Information } & $\begin{array}{l}\text { Sutton et al. [4], New } \\
\text { York }(n=215)\end{array}$ & $\begin{array}{l}\text { Vintzileos et al. [5], New } \\
\text { York }(n=161)\end{array}$ & $\begin{array}{l}\text { Gagliardi et al. [6], Tuscany } \\
\text { and Liguria }(n=533)\end{array}$ & $\begin{array}{l}\text { Our study, Madrid } \\
(n=203)\end{array}$ \\
\hline \multicolumn{2}{|c|}{$\begin{array}{l}\text { First obstetrical COVID-19 patient in the } \\
\text { city or region }\end{array}$} & March 3rd & March 3rd & February & February 25 th \\
\hline \multicolumn{2}{|c|}{ Lockdown ordered, date } & March 22nd & March 22nd & March 11th & March 14th \\
\hline \multicolumn{6}{|c|}{ Start of universal screening } \\
\hline \multicolumn{2}{|c|}{ Days after first case, $\mathrm{n}$} & 19 & 27 & Unknown & 36 \\
\hline \multicolumn{2}{|c|}{ Days after lockdown ordered, $\mathrm{n}$} & 0 & 8 & 15 & 23 \\
\hline \multirow{3}{*}{$\begin{array}{l}\text { Symptoms on } \\
\text { admission } \\
\text { Yes }\end{array}$} & $\begin{array}{l}\text { SARS-CoV-2 (qRT-PCR } \\
\text { test), n (\%) }\end{array}$ & & & & \\
\hline & Positive & $4(1.9)$ & $11(6.8)$ & $1(0.2)$ & $1(0.5)$ \\
\hline & Negative & $0(0)$ & $5(3.1)$ & $0(0)$ & $6(3.0)$ \\
\hline \multirow[t]{3}{*}{ No } & Positive & $29(13.5)$ & $21(13.0)$ & $2(0.4)$ & $1(0.5)$ \\
\hline & Negative & $181(84.2)^{\mathrm{a}}$ & $124(77.0)$ & $530(99.4)$ & $193(95.0)$ \\
\hline & Unknown & $1(0.5)$ & $0(0)$ & $0(0)$ & $2(1.0)$ \\
\hline
\end{tabular}

qRT-PCR, quantitative reverse transcription polymerase-chain-reaction. ${ }^{a}$ One case revealed positive postpartum.

case was diagnosed later but the screening started soon after this diagnosis and the lockdown, in Europe the situation was reversed. Our results and those of Tuscany and Liguria correlate with a significant and progressive reduction in the number of infections, the number of deceased and the circulation of new cases of COVID-19 in the community and almost only persisting symptomatic cases after a month of the start of confinement. However, the figures of around $13 \%$ found in New York correspond to the ascending phase of the pandemic when the effects of confinement were not yet evident.

Our experience also advises to adopt a proactive diagnostic management in admission of women for delivery since it provides security for her child, family members, other patients, and health staff. However, in the remission phase of the pandemic, new diagnostic strategies for pregnant women should be considered, such as those based on serological tests. These tests can be used as an adjuvant tool to detect SARS-CoV-2 when the viral titers and RT-PCR sensitivity decrease [7]. This could be of value to detect which women had SARS-CoV-2 during pregnancy in order to offer surveillance of their newborns, since it is currently unknown whether there is risk of vertical transmission and the long-term consequences [8].

In conclusion, the rate of positive results in asymptomatic women when universal qRT-PCR screening for SARS-CoV-2 is implemented at admission to labor - and therefore the effectiveness of this approach - depends on the circulation of new cases in the community. This is why caution is recommended when interpreting our results in areas with a different COVID-19 prevalence.
Research funding: None declared.

Author contributions: Ignacio Herraiz, conceptualization, data curation, formal analysis, writing original draft. Dolores Folgueira, conceptualization, review and editing. Cecilia Villalaín, data curation, review and editing. Laura Forcén, data curation, review and editing. Rafael Delgado, conceptualization, review and editing. Alberto Galindo, conceptualization, review and editing. All authors have accepted responsibility for the entire content of this manuscript and approved its submission.

Competing interests: Authors state no conflict of interest. Informed consent: Informed consent was obtained from all individuals included in this study.

Ethical approval: The study was approved by the local Research Ethics Committee. We have complied with the World Medical Association Declaration of Helsinki regarding ethical conduct of research involving human subjects.

Data availability statement: Research data are not shared but can be by request to the corresponding author.

\section{References}

1. Rasmussen SA, Smulian JC, Lednicky JA, Wen TS, Jamieson DJ. Coronavirus disease 2019 (COVID-19) and pregnancy: what obstetricians need to know. Am J Obstet Gynecol 2020;222: 415-26.

2. Corman VM, Landt O, Kaiser M, Molenkamp R, Meijer A, Chu DK, et al. Detection of 2019 novel coronavirus (2019-nCoV) by real-time RT-PCR. Euro Surveill 2020;25:2000045.

3. Cordes AK, Heim A. Rapid random access detection of the novel SARS-coronavirus-2 (SARS-CoV-2, previously 2019-nCoV) using an open 
access protocol for the Panther Fusion. J Clin Virol 2020;125:104305. https://doi.org/10.1016/j.jcv.2020.104305 [Epub ahead of print].

4. Sutton D, Fuchs K, D'Alton M, Goffman D. Universal screening for SARS-CoV-2 in women admitted for delivery. N Engl J Med 2020. https://doi.org/10.1056/NEJMc2009316 [Epub ahead of print].

5. Vintzileos WS, Muscat J, Hoffmann E, Vo D, John NS, Vertichio R, et al. Screening all pregnant women admitted to labor and delivery for the virus responsible for COVID-19. Am J Obstet Gynecol 2020. https://doi.org/10.1016/j.ajog.2020.04.024 [Epub ahead of print].

6. Gagliardi L, Danieli R, Suriano G, Vaccaro A, Tripodi G, Rusconi F, et al. Universal SARS-CoV-2 testing of pregnant women admitted for delivery in two Italian regions. Am J Obstet Gynecol 2020. https://doi.org/10.1016/j.ajog.2020.05.017 [Epub ahead of print].

7. Guo L, Ren L, Yang S, Xiao M, Chang D, Yang F, et al. Profiling early humoral response to diagnose novel coronavirus disease (COVID19). Clin Infect Dis 2020. https://doi.org/10.1093/cid/ciaa310 [Epub ahead of print].

8. Lamouroux A, Attie-Bitach T, Martinovic J, Leruez-Ville M, Ville Y. Evidence for and against vertical transmission for SARS-CoV-2 (COVID-19). Am J Obstet Gynecol 2020. https://doi.org/10.1016/j. ajog.2020.04.039 [Epub ahead of print]. 